\title{
APPLICATION OF AUTOLOGOUS SERUM EYE DROPS AFTER PTERYGIUM SURGERY
}

\author{
Hassan Sajjad Rathore, Ahsan Mukhtar, Shahzad Saeed, Umar Ijaz, Mamoona Javaid, Iqra Ghaus \\ Combined Military Hospital Quetta/National University of Medical Sciences (NUMS) Pakistan
}

\begin{abstract}
Objective: To compare the corneal epithelium healing time and post-operative pain after pterygium surgery with and without the use of autologous serum drops.

Study Design: Comparative cross-sectional study.

Place and Duration of Study: Eye Department, Combined Military Hospital Quetta, from Jan to Aug 2019.

Methodology: Twenty patients were included in this study and divided into two groups. All patients underwent pterygium excision with conjunctival autograft. Fourteen of them were instilled with autologous serum drops while 14 were given lubricants postoperatively until corneal epithelium healed completely. Corneal epithelium healing time, visual analog scale for postoperative pain assessment, conjunctival inflammation and recurrences were evaluated. Patients were followed up for 6 months.

Results: For corneal epithelial healing, 1 (7.14\%) patient showed complete healing on day 2 while 7 (50\%) showed healing on day 3 and $6(42.86 \%)$ on day 4 . While in non-autologous serum drops group $7(50 \%)$ showed complete healing on day 4 while $4(28.57 \%)$ showed healing on day 5 and $3(21.43 \%)$ on day 6 . The mean corneal healing time of autologous serum drops group was $3.36 \pm 0.63$ days and that of non-autologous serum drops group was $4.71 \pm 0.83$ days. Patients of autologous serum drops group also experienced significantly less pain as compared to the non-autologous serum drops group. While the recurrence rate and conjunctival inflammation in the two groups was found to be of equal degree.

Conclusion: Patients who used autologous serum eye drops post operatively experienced less pain and their corneal epithelium healed earlier than the non-autologous serum drops group.
\end{abstract}

Keywords: Autologous serum, Corneal epithelium, Pterygium.

How to Cite This Article: Rathore HS, Mukhtar A, Saeed S, Ijaz U, Javaid M, Ghaus I. Application of Autologous Serum Eye Drops After Pterygium Surgery. Pak Armed Forces Med J 2021; 71(5): 1563-1566. doi: https://doi.org/10.51253/pafmj.v71i5.3255

This is an Open Access article distributed under the terms of the Creative Commons Attribution License (http://creativecommons.org/licenses/by/4.0), which permits unrestricted use, distribution, and reproduction in any medium, provided the original work is properly cited.

\section{INTRODUCTION}

Pterygium is a benign, degenerative ocular disease, characterized by a fibrovascular growth invading the cornea from bulbar conjunctiva. It leads to significant tear film disturbance causing dry and irritable eyes, astigmatism and occasional severe visual deterioration if the visual axis is involved. ${ }^{1}$ It is usually present in regions with hot and humid climates, designated as the pterygium regions with temperatures ranging up to 30 degrees Celsius south and north of the equator. ${ }^{2}$ It typically presents in adults and in people with jobs related to more sunlight exposure due to outdoor activities. Other factors may be genetics, tumor suppressor gene $p 53$, male gender preponderance or a deficiency of limbal stem cells leading to conjunctivalization of the cornea. ${ }^{3,4}$

Prevalence of pterygium ranges from $0.7-31 \%$ in various studies around the world. ${ }^{5}$ In the province of Balochistan, Pakistan, a dry and arid climate prevails throughout the year. Extreme heat, dryness and dust

Correspondence: Dr Hasssan Sajjad Rathore, Eye Department, Combined Military Hospital, Quetta Pakistan

Received: 10 Sep 2019; revision received: 05 Jan 2020; accepted: 10 Jan 2020 storms are the main features of this climatic zone. In a recent study conducted by Shah et al, $86.63 \%$ of the total 1227-pterygium patients belonged to similar areas with hot and dry weather. ${ }^{6}$

Surgery is the main stay of treatment which has proven to give better results and improves the visual disturbances to the maximum. It is usually performed under local anesthetic infiltration. However, after the anesthetic effect wears off, post-operative pain and discomfort becomes the primary concern of the patient for the next 48-72 hours, contributing to patient dissatisfaction. Anesthetic agents, bandage contact lenses, NSAIDs and opioids have been used post operatively to reduce pain and discomfort. ${ }^{7}$

Autologous Serum Eye Drops (ASD) have been extensively used for ocular surface diseases, especially non-healing corneal ulcers. They have also been utilized to accelerate post-operative epithelial healing after refractive surgeries and keratoplasties. 8,9 In our search of the literature, very little local data was available that considered the effect of ASD on epithelial healing and post-operative pain after ptery-gium surgery. In this study, we planned to compare the corneal epithelium 
healing time and post-operative pain after pterygium surgery with and without the use of ASD.

\section{METHODOLOGY}

Ths study was conducted in the Eye department, Combined Military Hospital Quetta, from January to August 2019. It was a comparative cross-sectional study which was approved by the Ethics Committee of the institute (IERB no. Ext-23-05/READ-IRB/001) and informed written consent was taken from all patients. Inclusion criteria was all the pterygium patients presenting to the Eye Department.Patients were selected through universal sampling, irrespective of the age, gender, ethnicity and residence.

Exclusion criteria was patients with prior pterygium surgery, diabetes or any other ocular and systemic diseases leading to delayed corneal epithelium healing.

A proforma was used to record the personal profile of the patient and initial ocular examination, including visual acuity using Snellen's chart, intraocular pressure using applanation tonometry, detailed slit lamp anterior and posterior segment examination of both eyes.

Pterygium excision with conjunctival autograft was done in all the patients by the same surgeon, using similar technique. Cornea and conjunctiva were anesthetized by topical $0.5 \%$ proparacaine hydrochloride eye drops, supplemented by subconjunctival injection of $2 \%$ plain lignocaine hydrochloride. The head of the pterygium was dissected from the cornea with a crescent knife. Body of the pterygium with adjacent tenon's capsule was removed leading to bare sclera. Conjunctival graft from the harvest site was sutured onto the scleral bed in appropriate orientation with 7-0 vicryl sutures.

Block randomization was done using online research randomizer. Fourteen $(50 \%)$ out of the 28 patients were assigned to ASD group and the remaining were assigned to the non-ASD group according to generated random allocation sequence. Patients in the non-ASD group were given artificial tears (Dextran $0.1 \% \pm$ Hydroxypropyl Methylcellulose $0.3 \%$ ). Patients were advised to use ASD/artificial tears 4 hourly until the corneal epithelium healed completely. Remaining corneal epithelium defect was measured daily on a slit lamp, augmented by fluorescein staining. All of the patients also received antibiotic eye drops (tobramycin $0.3 \%$ ) which they used thrice a day for 2 weeks. After the surgery, post-operative pain was assessed using a visual analogue scale VAS (0-10) as shown in Figure-1.

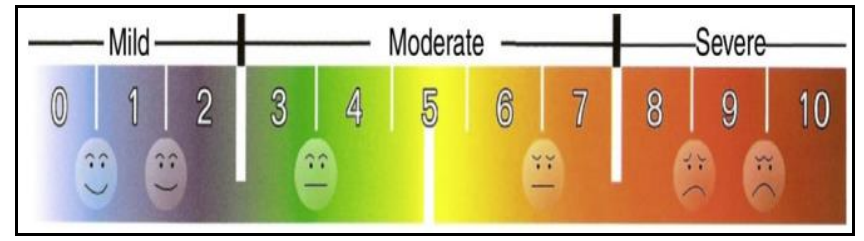

Figure-1: Visual analogue scale (VAS).

A score of 0 signified no pain and 10 signified worst pain. Recurrence was defined as emergence of any fibrovascular growth onto the cornea past the limbus. Conjunctival inflammation was defined as vascular dilation and hyperemia primarily in the host-conjunctival area. Conjunctival inflammation was graded as grade $0=$ no inflammation, grade $1=$ mild inflammation, grade $2=$ moderate inflammation, and grade $3=$ severe inflammation. All of the patients were examined daily at the same time for 6 days and then 1,2,3, and 6 months after surgery and epithelial defects were measured by the same examiner.

Whole blood $(15 \mathrm{ml})$ was taken from the vein (peripheral) of the patient and allowed to clot at room temperature for 1 hour. The blood was then centrifuged at 4000 RPM for 4 minutes. The serum was used in $100 \%$ concentration after centrifugation without any dilution. The serum drops were kept at 4 degree Celsius in refrigerator for use after surgery.

Data were analyzed using SPSS-23. Independent sample t-test was applied for the data variables. The $p$ value of $\leq 0.05$ was considered statistically significant.

\section{RESULTS}

Twenty eight eyes of 28 patients were included in the study. Patient with ages range of 24-47 years were randomly allocated into two groups, with 6 females $(21 \%)$ and 22 males $(79 \%)$. One group received ASD post-surgery while the other group received lubricant eye drops. The mean age of ASD group was 33.64 \pm 7.06 years and that of non-ASD group was $34.71 \pm$ 5.87 years.

For corneal epithelial healing $1(7.14 \%)$ out of the 14 patients who received ASD showed healing on day 2 while $7(50 \%)$ showed healing on day 3 and $6(42.86 \%)$ on day 4 . While in non-ASD group $7(50 \%)$ patients showed healing on day 4 while $4(28.57 \%)$ showed healing on day 5 and $3(21.43 \%)$ on day 6 (Figure-2).

Patients marked their pain perception postsurgery on VAS scale, from 0 being mild pain to 10 being severe pain. Patients of ASD group experienced a significantly lesser pain post-surgery due to early healing as shown in Table. 


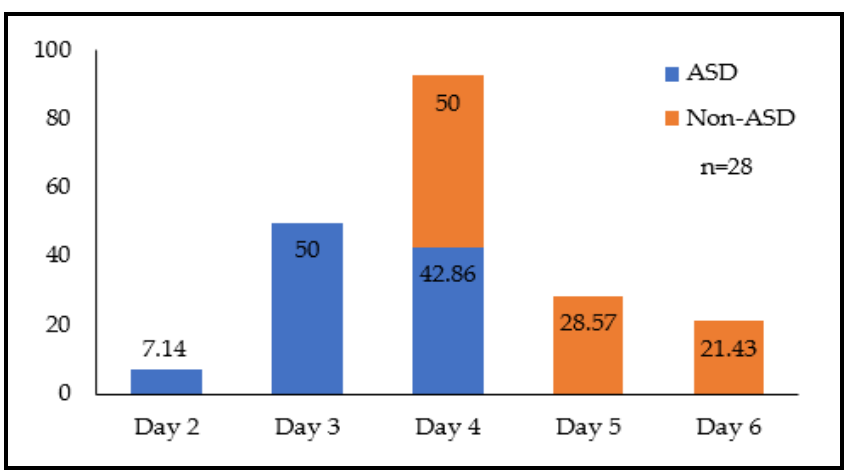

Figure-2: Corneal epithelium healing time. significant post-operative pain occurs in almost $60 \%$ of the patients, mostly on the $1^{\text {st }}$ post-operative day and slightly less on the $2^{\text {nd }}$ post-op day ${ }^{19}$ for which multiple techniques have been employed including topical anesthetics, NSAIDs and opioids. Long-term use of these medications may slow the epithelial healing leading to keratitis, sub epithelial infiltrates and thinning. Though the pain reduction is reported to be efficient, yet prolonged use may lead to complications as mentioned. ${ }^{19,20}$ Bandage contact lenses have also been used to reduce pain as well as for epithelial healing. Yeung et al, reported in their study that bandage contact lens

Table: Visual analogue scale for post-operative pain.

\begin{tabular}{l|c|c|c}
\hline Days & Autologous Serum Drops Group & Non-Autologous Serum Drops Group & $p$-value \\
\hline Visual Analogue Scale Day 1 & $4.71 \pm 0.91$ & $7.36 \pm 1.39$ & $<0.001$ \\
\hline Visual Analogue Scale Day 2 & $3.07 \pm 0.73$ & $5.07 \pm 1.49$ & $<0.001$ \\
\hline Visual Analogue Scale Day 3 & $1.42 \pm 0.94$ & $2.79 \pm 1.12$ & 0.002 \\
\hline Visual Analogue Scale Day 4 & $0.36 \pm 0.50$ & $1.29 \pm 0.91$ & 0.003 \\
\hline Visual Analogue Scale Day 5 & $0.14 \pm 0.36$ & $0.50 \pm 0.52$ & 0.045 \\
\hline
\end{tabular}

\section{DISCUSSION}

Even though surgery is the gold standard treatment for pterygium, yet recurrence remains the most unwanted outcome after successful surgery. The older "Bare Sclera" technique, which was widely used, has been almost abandoned due to high recurrence rates. Other modalities include application of strontium 90, beta irradiation, mitomycin-C and 5-flourouracil application. ${ }^{10,11}$ In a review by Clearfield et al, conjunctival autografts came out to be a better surgical option with a lower recurrence rate of about $16.7 \%$ than recurrence rate of amniotic membrane graft of about $47 \% .{ }^{12}$ Various techniques employed in grafting include the use of sutures, fibrin glue, autologous serum drops and electrocautery. ${ }^{13}$ Pterygium excision with conjunctival autograft is, thus, the most effective technique used for pterygia removal, in terms of pain post-surgery and the recurrence rate. ${ }^{14,15} \mathrm{It}$ is believed that pterygium recurrence is caused by the surgical trauma and subsequent postoperative inflammation, leading to activation and proliferation of subconjunctival fibroblasts and vascular cells. ${ }^{16}$

ASD may be a triggering factor for ocular inflammation for it has pro-inflammatory cytokines (IL-7 and INF- $\gamma$ ) and other metalloproteinases. However, our study revealed there was no significant difference in ocular inflammation post-surgery with or without the use of ASD, 17,18 (both groups had moderate conjunctival inflammation). Pain after pterygium surgery has always been of great concern causing patient dissatisfaction post-surgery. Wishaw et al, demonstrated that is one of the effective ways in provision of considerable pain relief and overnight wear helps to prevent the agonizing pain post-surgery, ${ }^{21}$ but they may induce bacterial keratitis in eyes without the protection of an epithelial barrier.

The corneal epithelial defect made during the surgery, is the main cause of ocular pain and increases the susceptibility to different infections. Therefore, in our study, we used autologous serum eye drops to accelerate the closing of this epithelial defect. In a similar study carried out in Turkey, Sul et al, also used autologous serum post pterygium excision. ${ }^{18}$ Although they had similar conjunctival inflammation scores and recurrence rates, yet their corneal epithelium healing time and post-operative pain scores were slightly better than our results. The reasons of this disparity may be use of different concentration of autologous serum, different sample population or different surgical technique. Pan et al, reflect in their article that autologous serum drops offer a potential advantageous role in acting as a tear substitute and contains components that help in mimicking the beneficial effects of tears in cases like epithelial diseases and dry eyes. ${ }^{22}$ According to Lopez-Garcia, autologous serum is rich in growth factors, nutrients and healing agents which help to induce healing and promote the wound closure postsurgery. ${ }^{23}$ However, the use of ASD after pterygium surgery has not been reported sufficiently in the literature, especially in our population.

Different problem areas have also been identified in using autologous serum, including contamination of 
serum leading to keratitis, denaturation of serum if not kept in appropriate temperature, an increase in ocular inflammation and the added burden of an additional procedure for withdrawing $15-20 \mathrm{ml}$ of extra blood from every patient. Therefore, these problems should also be catered for before using autologous serum. Although our study has some limitations like the lack of diversity in sample size, the importance of our study lies in inclusion of both subjective and objective data in calculating the results. However, we do need more studies with a more diverse sample to confirm the efficacy of autologous serum in pterygium surgeries.

\section{CONCLUSION}

Autologous serum was a good option that helped in promoting early healing and pain relief in patients post pterygium surgery, which in turn increased patient satisfaction.

\section{Conflict of Interest: None.}

\section{Authors' Contribution}

HSR: Conception \& manuscripts writing, AM: Manuscripts writing \& data collection, SS: Manuscripts writing \& referencing, UI: Design \& interpretation of data, MJ: Manuscripts writing \& statistical analysis, IG: Data collection \& proof reading.

\section{REFERENCES}

1. Singh SK. Pterygium: epidemiology prevention and treatment. Community Eye Health 2017; 30(99): S5-S10.

2. Matthew H, Chui Jeanie J, Tat Lien. Significance of fuchs flecks in patients with pterygium/pinguecula: earliest indicator of ultra-violet light damage. Cornea 2015; 34(12): 1560-1563.

3. Kaufman SC, Jacobs DS, Lee WB, Deng SX. Options and adjuvants in surgery for pterygium: a report by the American Academy of Ophthalmology. Ophthalmol 2013; 120(1): 201-208.

4. Hall $\mathrm{AB}$. Understanding and managing pterygium. Community Eye Health 2016; 29(95): 54-56.

5. Alpay A, Uğurbaş SH, Erdoğan B. Comparing techniques for pterygium surgery. Clin Ophthalmol 2009; 2009(3): 69-74.

6. Shah SI, Shah SA, Rai P. Factors associated with pterygium based on history and clinical examination of patients in Pakistan. J Curr Ophthalmol 2016; 28(2): 91-92.

7. Moussa K, Shantha J. Blurry vision and eye pain after pterygium surgery. JAMA Ophthalmol 2018; 136(7): 827-828.

8. Chen Y, Hu F, Huang J, Shen EP, Tsai TY, Chen WL. The effect of topical autologous serum on graft re-epithelialization after penetrating keratoplasty. Am J Ophthalmol 2010; 150(3): 352-359.
9. Hondur AM, Akcam T, Karaca EE, Yazici Eroglu H, Aydin B. Autologous serum eye drops accelerate epithelial healing after LASEK. Curr Eye Res 2016; 41(1): 15-19.

10. Santos Martins TG, de Azevedo Costa AL, Alves MR, Chammas $\mathrm{R}$, Schor P. Mitomycin C in pterygium treatment. Int J Ophthalmol 2016; 9(3): 465-468.

11. Bekibele CO, Sarimiye TF, Ogundipe A, Olaniyan S. 5Fluorouracil vs Avastin as adjunct to conjunctival autograft in the surgical treatment of pterygium. Eye 2016; 30(4): 515521.

12. Clearfield E, Hawkins BS, Kuo IC. Conjunctival autograft versus amniotic membrane transplantation for treatment of pterygium: findings from a cochrane systematic review. Am J Ophthalmol 2017; 182(2): 8-17.

13. Han SB, Jeon HS, Kim M, Lee SJ, Yang HK, Hwang JM, et al.Risk factors for recurrence after pterygium surgery: an image analysis study. Cornea 2016; 35(8): 1097-1103.

14. Hacioglu D. Developments and current approaches in the treatment of pterygium. Int Ophthalmol 2017; 37(4): 1073-1081.

15. Salman AG, Mansour DE. The recurrence of pterygium after different modalities of surgical treatment. Saudi J Ophthalmol 2011; 25(4): 411-415.

16. Ma DK, See LC, Liau SB. Amniotic membrane graft for primary pterygium: comparison with conjunctival autograft and topical mitomycin C treatment. Br J Ophthalmol 2000; 84(9): 973-978.

17. Ha SW, Park JH, Im Hee Shin HK. Clinical analysis of risk factors contributing to recurrence of pterygium after excision and graft surgery. Inter J Ophthalmol 2015; 8(3): 522-527.

18. Sul S, Korkmaz S, Alacamli G. Application of autologous serum eye drops after pterygium surgery: a prospective study. Graefes Arch Clin Exp Ophthalmol 2018; 256(10): 1939-1943.

19. Wishaw K, Billington D, O' Brien D, Davies P. The use of orbital morphine for postoperative analgesia in pterygium surgery. Anaes Intens Care 2000; 28(1): 43-45.

20. Iwamoto S, Koga T, Ohba M, Okuno T, Koike M, Murakami A, et al. Non-steroidal anti-inflammatory drug delays corneal wound healing by reducing production of 12-hydroxyheptadecatrienoic acid, a ligand for leukotriene B4 receptor 2. Sci Rep 2017; 7(1): 13267-13274.

21. Yeung SN, Lichtinger A, Kim P, Elbaz U, Ku JY, Teichman JC, et al. Efficacy and safety of patching vs bandage lens on postoperative pain following pterygium surgery. Eye 2015; 29(2): 295-296.

22. Pan Q, Angelina A, Marrone M, Stark WJ, Akpek EK. Autologous serum eye drops for dry eye. Cochrane Database Syst Rev 2017; 2(2): CD009327.

23. López-García JS, García-Lozano I, Rivas L, Giménez C, Acera A, Suárez-Cortés T. Effects of autologous serum eye drops on conjunctival expression of MUC5AC in patients with ocular surface disorders. Cornea 2016; 35(3): 336-341. 